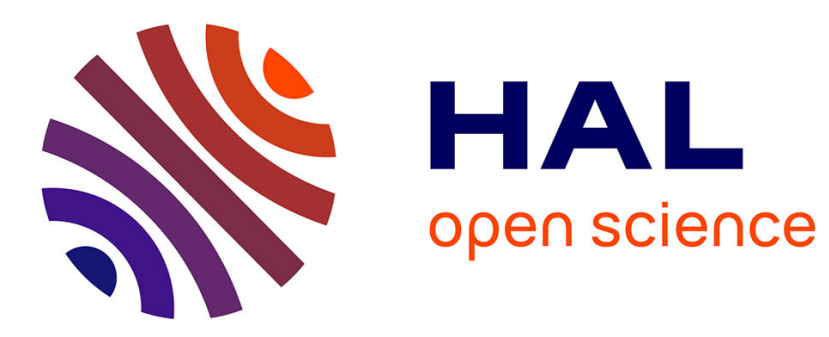

\title{
Kernel estimators of extreme level curves
}

Abdelaati Daouia, Laurent Gardes, Stéphane Girard, Alexandre Lekina

\section{To cite this version:}

Abdelaati Daouia, Laurent Gardes, Stéphane Girard, Alexandre Lekina. Kernel estimators of extreme level curves. Test, 2011, 20 (2), pp.311-333. 10.1007/s11749-010-0196-0 . inria-00393588v4

\section{HAL Id: inria-00393588 https://hal.inria.fr/inria-00393588v4}

Submitted on 23 Apr 2013

HAL is a multi-disciplinary open access archive for the deposit and dissemination of scientific research documents, whether they are published or not. The documents may come from teaching and research institutions in France or abroad, or from public or private research centers.
L'archive ouverte pluridisciplinaire HAL, est destinée au dépôt et à la diffusion de documents scientifiques de niveau recherche, publiés ou non, émanant des établissements d'enseignement et de recherche français ou étrangers, des laboratoires publics ou privés. 


\title{
Kernel estimators of extreme level curves
}

\author{
Abdelaati Daouia ${ }^{(1)}$, Laurent $\operatorname{Gardes}^{(2)}$, Stéphane Girard ${ }^{(2, \star)} \&$ Alexandre Lekina ${ }^{(2)}$ \\ (1) Toulouse School of Economics (GREMAQ), University of Toulouse, Aile J.J. \\ Laffont, 21 allée de Brienne, 31000 Toulouse, France. \\ (2) INRIA Rhône-Alpes, team Mistis, Inovallée, 655, av. de l'Europe, Montbonnot, \\ 38334 Saint-Ismier cedex, France. \\ (^) Stephane.Girard@inrialpes.fr (corresponding author)
}

\begin{abstract}
We address the estimation of extreme level curves of heavy-tailed distributions. This problem is equivalent to estimating quantiles when covariate information is available and when their order converges to one as the sample size increases. We show that, under some conditions, these so-called "extreme conditional quantiles" can still be estimated through a kernel estimator of the conditional survival function. Sufficient conditions on the rate of convergence of their order to one are provided to obtain asymptotically Gaussian distributed estimators. Making use of this result, some kernel estimators of the conditional tail-index are introduced and a Weissman type estimator is derived, permitting to estimate extreme conditional quantiles of arbitrary large order. These results are illustrated through simulated and real datasets.
\end{abstract}

Keywords: Conditional quantiles, heavy-tail distributions, kernel estimator, extreme-values. AMS 2000 subject classification: 62G32, 62G30, 62E20.

\section{Introduction}

Let $\left(X_{i}, Y_{i}\right), i=1, \ldots, n$ be independent copies of a random pair $(X, Y)$ in $\mathbb{R}^{p} \times \mathbb{R}$. We address the problem of estimating extreme level curves, defined as the graphs of the functions $x \in \mathbb{R}^{p} \mapsto q\left(\alpha_{n} \mid x\right) \in \mathbb{R}$ verifying $\mathbb{P}\left(Y>q\left(\alpha_{n} \mid x\right) \mid X=x\right)=\alpha_{n}$ where $\alpha_{n} \rightarrow 0$ as $n \rightarrow \infty$. In such a case, $q\left(\alpha_{n} \mid x\right)$ is referred to as an extreme conditional quantile in contrast to classical conditional quantiles (known as regression quantiles) for which $\alpha_{n}=\alpha$ is fixed in $(0,1)$. While the nonparametric estimation of ordinary regression quantiles has been extensively studied (see for instance the seminal papers $[37,40]$ or [15], Chapter 5), less attention has been paid to extreme conditional quantiles despite their potential interest. In the financial econometrics literature, one will to understand the extreme behavior between hedge fund returns and measures of risk. Here 
the vector $X$ represents the set of $p$ risk factors (volatility or variance, kurtosis, ...) and the variable $Y$ is a returns measure (average return, skewness, ...). Hedge fund returns may exhibit particular characteristics such as high degree of non-normality and fat tails that need to be incorporated into the estimation procedure. Applications in the finance field include also work on Value at Risk, option pricing and the analysis of cross section of stock market returns (see, e.g., [34]). In climatology, one may be interested in how climate change over years might affect extreme temperatures. Here, the covariate is univariate (the time). Multivariate examples include the study of extreme rainfall as a function of the geographical location. Parametric models are proposed in $[11,39]$ whereas semi-parametric methods are considered in [3, 29]. Fully non-parametric estimators have been first introduced in [10], where a local polynomial modeling of the extreme observations is used. Similarly, spline estimators are fitted in [8] through a penalized maximum likelihood method. In both cases, the authors focus on univariate covariates and on the finite sample properties of the estimators. An important literature is devoted to the particular case where the conditional distribution of $Y$ given $X=x$ has a finite endpoint $\varphi(x)$. The function $\varphi$ is referred to as the frontier and can be estimated via an estimator of the conditional quantile $q\left(\alpha_{n} \mid x\right)$ with $\alpha_{n} \rightarrow 0$. As an example, a kernel estimator of $\varphi$ is proposed in [25] with $\alpha_{n}=1 / n$, the asymptotic normality being proved only in the situation where $Y$ given $X=x$ is uniformly distributed on $[0, \varphi(x)]$. In this latter situation, regression on the extreme values of the sample has also been introduced in $[18,24,26,35]$, the case where the density of $Y$ given $X=x$ is lower bounded being first studied by Geffroy [22]. Extensions are provided in [23, 30, 31] to densities of $Y$ given $X=x$ decreasing as a power of the distance from the boundary. We refer to [33] for more information on this topic.

Estimation of unconditional extreme quantiles is also widely studied since the introduction of Weissman estimator [42], dedicated to heavy tail distributions, and Dekkers and de Haan estimator [12] adapted to the general case.

In this paper, we focus on the setting where the conditional distribution of $Y$ given $X=x$ has an infinite endpoint and is heavy-tailed, an analytical characterization of this property being given in the next section. In such a case, the frontier function does not exist and $q\left(\alpha_{n} \mid x\right) \rightarrow \infty$ as $\alpha_{n} \rightarrow 0$. Nevertheless, we show, under some mild conditions, that extreme regression quantiles $q\left(\alpha_{n} \mid x\right)$ can still be estimated through a kernel estimator of $\mathbb{P}(Y>. \mid x)$. We provide sufficient conditions on the rate of convergence of $\alpha_{n}$ to 0 so that our estimator is asymptotically Gaussian distributed. Making use of this, some kernel estimators of the conditional tail-index are introduced and a Weissman type estimator [42] is derived, permitting to estimate extreme conditional quantiles $q\left(\beta_{n} \mid x\right)$ where $\beta_{n} \rightarrow 0$ arbitrarily fast.

Assumptions are introduced and discussed in Section 2. Our main results are provided in Section 3 and illustrated on simulated data in Section 4. An example of application to real data is presented in Section 5. Proofs are postponed to the appendix. 


\section{Notations and assumptions}

The conditional survival function (csf) of $Y$ given $X=x$ is denoted by $\bar{F}(y \mid x)=\mathbb{P}(Y>y \mid X=x)$ and the probability density function (pdf) of $X$ is denoted by $g$. The kernel estimator of $\bar{F}(y \mid x)$ is defined for all $(x, y) \in \mathbb{R}^{p} \times \mathbb{R}$ by

$$
\hat{\bar{F}_{n}}(y \mid x)=\sum_{i=1}^{n} K_{h}\left(x-X_{i}\right) \mathbb{I}\left\{Y_{i}>y\right\} / \sum_{i=1}^{n} K_{h}\left(x-X_{i}\right),
$$

where $\mathbb{I}\{$.$\} is the indicator function and h=h_{n}$ is a nonrandom sequence such that $h \rightarrow 0$ as $n \rightarrow \infty$. We have also introduced $K_{h}(t)=K(t / h) / h^{p}$ where $K$ is a pdf on $\mathbb{R}^{p}$. In this context, $h$ is called the window-width. In Theorem 1, the asymptotic distribution of (1) is established when estimating small tail probabilities, i.e when $y=y_{n}$ goes to infinity with the sample size $n$. Similarly, the kernel estimators of conditional quantiles $q(\alpha \mid x)$ are defined via the generalized inverse of $\hat{\bar{F}}_{n}(. \mid x)$ :

$$
\hat{q}_{n}(\alpha \mid x)=\hat{\bar{F}}_{n}^{\leftarrow}(\alpha \mid x)=\inf \left\{t, \hat{\bar{F}}_{n}(t \mid x) \leq \alpha\right\},
$$

for all $\alpha \in(0,1)$. Many authors are interested in this type of estimator for fixed $\alpha \in(0,1)$ : weak and strong consistency are proved respectively in [40] and [16], asymptotic normality being established in [41, 38, 4]. In Theorem 2, the asymptotic distribution of (2) is investigated when estimating extreme quantiles, i.e when $\alpha=\alpha_{n}$ goes to 0 as the sample size $n$ goes to infinity. The asymptotic behavior of such estimators depends on the nature of the conditional distribution tail. In this paper, we focus on heavy tails. More specifically, we assume that the csf satisfies

(F.1): $\bar{F}(y \mid x)=y^{-1 / \gamma(x)} \ell(y \mid x)$,

where $\gamma($.$) is a positive function of the covariate x$ and, for $x$ fixed, $\ell(. \mid x)$ is a slowly-varying function at infinity, i.e for all $\lambda>0$,

$$
\lim _{y \rightarrow \infty} \frac{\ell(\lambda y \mid x)}{\ell(y \mid x)}=1
$$

To summarize, (F.1) amounts to assuming that the conditional distribution of $Y$ given $X=x$ is in the Fréchet maximum domain of attraction. In this context, $\gamma(x)$ is referred to as the conditional tail-index since it tunes the tail heaviness of the conditional distribution of $Y$ given $X=x$. Assumption (F.1) is also equivalent to stating that $\bar{F}(. \mid x)$ is regularly varying at infinity with index $-1 / \gamma(x)$. As remarked in [7], p.15, one can assume that

(F.2): $\ell(. \mid x)$ is normalized,

without loosing generality since slowly-varying functions are of interest only asymptotically. In such a case, the Karamata representation (see [7], Theorem 1.3.1) of the slowly-varying function can be written as

$$
\ell(y \mid x)=c(x) \exp \left(\int_{1}^{y} \frac{\varepsilon(u \mid x)}{u} d u\right)
$$

where $c($.$) is a positive function and \varepsilon(y \mid x) \rightarrow 0$ as $y \rightarrow \infty$. Thus, $\ell(. \mid x)$ is differentiable and the auxiliary function is given by $\varepsilon(y \mid x)=y \ell^{\prime}(y \mid x) / \ell(y \mid x)$. This function plays an important role in 
extreme-value theory since it drives the speed of convergence in (3) and more generally the bias of extreme-value estimators. Therefore, it may be of interest to specify how it converges to 0 . In $[1,2,27]$, the auxiliary function is supposed to be regularly varying and the estimation of the corresponding regular variation index is addressed. Here, we limit ourselves to assuming that

(F.3): $|\varepsilon(. \mid x)|$ is continuous and ultimately non-increasing.

Some Lipschitz conditions are also required. For all $\left(x, x^{\prime}\right) \in \mathbb{R}^{p} \times \mathbb{R}^{p}$, the Euclidean distance between $x$ and $x^{\prime}$ is denoted by $d\left(x, x^{\prime}\right)$ and the following assumptions are introduced:

(L.1): There exists $c_{\gamma}>0$ such that $\left|\frac{1}{\gamma(x)}-\frac{1}{\gamma\left(x^{\prime}\right)}\right| \leq c_{\gamma} d\left(x, x^{\prime}\right)$.

(L.2): There exist $c_{\ell}>0$ and $y_{0}>1$ such that $\sup _{y \geq y_{0}}\left|\frac{\log \ell(y \mid x)}{\log y}-\frac{\log \ell\left(y \mid x^{\prime}\right)}{\log y}\right| \leq c_{\ell} d\left(x, x^{\prime}\right)$.

(L.3): There exists $c_{g}>0$ such that $\left|g(x)-g\left(x^{\prime}\right)\right| \leq c_{g} d\left(x, x^{\prime}\right)$.

The last assumption is standard in the kernel estimation framework.

(K): $K$ is a bounded pdf on $\mathbb{R}^{p}$, with support $S$ included in the unit ball of $\mathbb{R}^{p}$.

We refer to $[19,20,21]$ for a similar model in the fixed-design setting.

\section{Main results}

Let us first focus on the estimation of small tail probabilities $\bar{F}\left(y_{n} \mid x\right)$ when $y_{n} \rightarrow \infty$ as $n \rightarrow \infty$. The following result provides sufficient conditions for the asymptotic normality of $\hat{\bar{F}}_{n}\left(y_{n} \mid x\right)$.

Theorem 1 Suppose (F.1), (L.1), (L.2), (L.3) and (K) hold. Let us introduce

- $0<a_{1}<a_{2}<\cdots<a_{J}$ where $J$ is a positive integer,

- $y_{n} \rightarrow \infty$ such that $n h^{p} \bar{F}\left(y_{n} \mid x\right) \rightarrow \infty$ and $n h^{p+2} \log ^{2}\left(y_{n}\right) \bar{F}\left(y_{n} \mid x\right) \rightarrow 0$ as $n \rightarrow \infty$,

- $y_{n, j}=a_{j} y_{n}$ for $j=1, \ldots, J$.

Then, for all $x \in \mathbb{R}^{p}$ such that $g(x)>0$, the random vector

$$
\left\{\sqrt{n h^{p} \bar{F}\left(y_{n} \mid x\right)}\left(\frac{\hat{\bar{F}}_{n}\left(y_{n, j} \mid x\right)}{\bar{F}\left(y_{n, j} \mid x\right)}-1\right)\right\}_{j=1, \ldots, J}
$$

is asymptotically Gaussian, centered, with covariance matrix $\frac{\|K\|_{2}^{2}}{g(x)} C(x)$ where $C_{j, j^{\prime}}(x)=a_{j \wedge j^{\prime}}^{1 / \gamma(x)}$ for $\left(j, j^{\prime}\right) \in\{1, \ldots, J\}^{2}$.

Note that $n h^{p} \bar{F}\left(y_{n} \mid x\right) \rightarrow \infty$ is a necessary and sufficient condition for the almost sure presence of at least one sample point in the region $B(x, h) \times\left(y_{n}, \infty\right)$ of $\mathbb{R}^{p+1}$, where $B(x, h)$ is the ball centered at $x$ with radius $h$. See Lemma 3 in Appendix. Thus, this natural condition states that one cannot estimate small tail probabilities out of the sample using $\hat{\bar{F}}_{n}$. This result may be compared to [13] which establishes the asymptotic behavior of the empirical survival function in the unconditional case but without assumption on the distribution. Considering now the estimation 
of extreme quantiles $q\left(\alpha_{n} \mid x\right)$ when $\alpha_{n} \rightarrow 0$ as $n \rightarrow \infty$, the asymptotic normality of $\hat{q}_{n}\left(\alpha_{n} \mid x\right)$ can be established under similar conditions.

Theorem 2 Suppose (F.1), (F.2), (L.1), (L.2), (L.3) and (K) hold. Let us introduce

- $\tau_{1}>\tau_{2}>\cdots>\tau_{J}>0$ where $J$ is a positive integer,

- $\alpha_{n} \rightarrow 0$ such that $n h^{p} \alpha_{n} \rightarrow \infty$ and $n h^{p+2} \alpha_{n} \log ^{2}\left(\alpha_{n}\right) \rightarrow 0$ as $n \rightarrow \infty$,

- $\alpha_{n, j}=\tau_{j} \alpha_{n}$ for $j=1, \ldots, J$.

Then, for all $x \in \mathbb{R}^{p}$ such that $g(x)>0$, the random vector

$$
\left\{\sqrt{n h^{p} \alpha_{n}}\left(\frac{\hat{q}_{n}\left(\alpha_{n, j} \mid x\right)}{q\left(\alpha_{n, j} \mid x\right)}-1\right)\right\}_{j=1, \ldots, J}
$$

is asymptotically Gaussian, centered, with covariance matrix $\|K\|_{2}^{2} \frac{\gamma^{2}(x)}{g(x)} \Sigma$ where $\Sigma_{j, j^{\prime}}=1 / \tau_{j \wedge j^{\prime}}$ for $\left(j, j^{\prime}\right) \in\{1, \ldots, J\}^{2}$.

Compared to [4], Theorem 6.4 , where $\alpha_{n}=\alpha$ is fixed in $(0,1)$, the asymptotic variance is larger in Theorem 2 since it involves the additional term $1 / \alpha_{n} \rightarrow \infty$ as $n \rightarrow \infty$. Let us also highlight the important role of the conditional tail-index $\gamma(x)$. From the asymptotic variance point of view, our model is equivalent to a csf with constant tail-index 1 and a pdf proportional to $g(x) / \gamma^{2}(x)$. In such a case, the number of points in the ball $B(x, h)$ is asymptotically inversely proportional to $\gamma^{2}(x)$. A large value of the tail-index at $x$ thus implies a difficult estimation of $q\left(\alpha_{n} \mid x\right)$.

Remark 1 Clearly, the condition $n h^{p} \alpha_{n} \rightarrow \infty$ implies $h>\left(n \alpha_{n}\right)^{-1 / p}$ eventually. Replacing in condition $n h^{p+2} \alpha_{n} \log ^{2}\left(\alpha_{n}\right) \rightarrow 0$ yields $n \alpha_{n} \log ^{-p}\left(1 / \alpha_{n}\right) \rightarrow \infty$ which entails $\alpha_{n}>\log ^{p}(n) / n$ eventually. This condition provides a lower bound on the order of the extreme quantiles for the asymptotic normality of kernel estimators to hold.

Remark 2 Suppose $n \alpha_{n} \log ^{2}\left(\alpha_{n}\right) \rightarrow \infty$ as $n \rightarrow 0$. To fulfill the assumptions of Theorem 2, one can choose $h=\eta_{n}\left(n \alpha_{n} \log ^{2}\left(\alpha_{n}\right)\right)^{-1 /(p+2)}$ where $\left(\eta_{n}\right)$ is a sequence tending to zero arbitrarily slowly. This choice yields an asymptotic variance proportional to $\frac{1}{n h^{p} \alpha_{n}}=\eta_{n}^{-p}\left(\frac{n \alpha_{n}}{\log ^{p}\left(\alpha_{n}\right)}\right)^{-2 /(p+2)}$. Note that, for $p=0$, we find back the variance of estimators dedicated to unconditional extreme quantiles.

A kernel version of Pickands estimator [36] for the conditional tail-index $\gamma(x)$ can be proposed on the basis of $\hat{q}_{n}(. \mid x)$, the kernel estimator defined in (2):

$$
\hat{\gamma}_{n}^{\mathrm{P}}(x)=\frac{1}{\log 2} \log \left(\frac{\hat{q}_{n}\left(\alpha_{n} \mid x\right)-\hat{q}_{n}\left(2 \alpha_{n} \mid x\right)}{\hat{q}_{n}\left(2 \alpha_{n} \mid x\right)-\hat{q}_{n}\left(4 \alpha_{n} \mid x\right)}\right),
$$

where $\alpha_{n} \rightarrow 0$ as $n \rightarrow \infty$. We refer to [23] for a different variant of Pickands estimator in the context where the distribution of $Y$ given $X=x$ has a finite endpoint. Letting $\sigma_{n}=\left(n h^{p} \alpha_{n}\right)^{-1 / 2}$, the asymptotic normality of $\hat{\gamma}_{n}^{\mathrm{P}}(x)$ is a consequence of Theorem 2 . 
Corollary 1 Suppose (F.1), (F.2), (F.3), (L.1), (L.2), (L.3) and (K) hold. If $\sigma_{n} \rightarrow 0$, $\sigma_{n}^{-1} h \log \alpha_{n} \rightarrow 0$ and $\sigma_{n}^{-1} \varepsilon\left(q\left(2 \alpha_{n} \mid x\right) \mid x\right) \rightarrow 0$ as $n \rightarrow \infty$, then, for all $x \in \mathbb{R}^{p}$ such that $g(x)>0$, $\sigma_{n}^{-1}\left(\hat{\gamma}_{n}^{P}(x)-\gamma(x)\right)$ converges to a centered Gaussian random variable with variance

$$
\frac{\|K\|_{2}^{2}}{g(x)} \frac{\gamma^{2}(x)\left(2^{2 \gamma(x)+1}+1\right)^{2}}{4(\log 2)^{2}\left(2^{\gamma(x)}-1\right)^{2}} .
$$

It should be clear that (5) is, up to the scale factor $\|K\|_{2}^{2} / g(x)$, the variance of the classical Pickands estimator, see for instance [28], Theorem 3.3.5. Since this variance is huge for large values of the conditional tail-index, it may be preferable to consider a kernel version of the Hill estimator [32]:

$$
\hat{\gamma}_{n}^{\mathrm{H}}(x)=\sum_{j=1}^{J}\left[\log \hat{q}_{n}\left(\tau_{j} \alpha_{n} \mid x\right)-\log \hat{q}_{n}\left(\alpha_{n} \mid x\right)\right] / \sum_{j=1}^{J} \log \left(1 / \tau_{j}\right),
$$

where $\left(\tau_{j}\right)$ is a decreasing sequence of weights.

Corollary 2 Suppose (F.1), (F.2), (F.3), (L.1), (L.2), (L.3) and (K) hold. Let $1=\tau_{1}>\tau_{2}>$ $\cdots>\tau_{J}>0$ where $J$ is a positive integer. If $\sigma_{n} \rightarrow 0, \sigma_{n}^{-1} h \log \alpha_{n} \rightarrow 0$ and $\sigma_{n}^{-1} \varepsilon\left(q\left(\alpha_{n} \mid x\right) \mid x\right) \rightarrow 0$ as $n \rightarrow \infty$, then, for all $x \in \mathbb{R}^{p}$ such that $g(x)>0, \sigma_{n}^{-1}\left(\hat{\gamma}_{n}^{H}(x)-\gamma(x)\right)$ converges to a centered Gaussian random variable with variance $\|K\|_{2}^{2} \gamma^{2}(x) V_{J} / g(x)$ where

$$
V_{J}=\left(\sum_{j=1}^{J} \frac{2(J-j)+1}{\tau_{j}}-J^{2}\right) /\left(\sum_{j=1}^{J} \log \left(1 / \tau_{j}\right)\right)^{2} .
$$

As an example, choosing $\tau_{j}=1 / j$ for each $j=1, \ldots, J$ yields $V_{J}=J(J-1)(2 J-1) /\left(6 \log ^{2}(J !)\right)$. In this case, $V_{J}$ is a convex function of $J$ and is minimum for $J=9$ leading to $V_{9} \simeq 1.25$. Moreover, introducing

$$
\hat{g}_{n}(x)=\frac{1}{n} \sum_{i=1}^{n} K_{h}\left(x-X_{i}\right)
$$

the classical kernel estimator of the pdf $g(x)$, one may obtain pointwise confidence intervals for extreme conditional quantiles by plugging $\hat{g}_{n}(x)$ and $\hat{\gamma}_{n}^{\mathrm{P}}(x)$ or $\hat{\gamma}_{n}^{\mathrm{H}}(x)$ in the asymptotic covariance matrix. Indeed, all estimates are consistent, as shown in Lemma 4, Corollary 1 and Corollary 2. As pointed out in Remark 1, the kernel estimator of extreme quantiles $\hat{q}_{n}\left(\alpha_{n} \mid x\right)$ requires a stringent condition on the order $\alpha_{n}$ of the quantile, since by construction it cannot extrapolate beyond the maximum observation in the ball $B(x, h)$. To overcome this limitation, a Weissman type estimator [42] can be derived:

$$
\hat{q}_{n}^{\mathrm{W}}\left(\beta_{n} \mid x\right)=\hat{q}_{n}\left(\alpha_{n} \mid x\right)\left(\alpha_{n} / \beta_{n}\right)^{\hat{\gamma}_{n}(x)}
$$

Here, $\hat{q}_{n}\left(\alpha_{n} \mid x\right)$ is the kernel estimator of the extreme quantile considered so far and $\hat{\gamma}_{n}(x)$ is an estimator of the conditional tail-index $\gamma(x)$. As illustrated in the next theorem, the extrapolation factor $\left(\alpha_{n} / \beta_{n}\right)^{\hat{\gamma}_{n}(x)}$ allows to estimate extreme quantiles of order $\beta_{n}$ arbitrary small. 
Theorem 3 Suppose (F.1), (F.2), (L.1), (L.2), (L.3) and (K) hold. Let us introduce

- $\alpha_{n} \rightarrow 0$ such that $\sigma_{n} \rightarrow 0$ and $\sigma_{n}^{-1} h \log \alpha_{n} \rightarrow 0$ as $n \rightarrow \infty$,

- $\left(\beta_{n}\right)$ such that $\beta_{n} / \alpha_{n} \rightarrow 0$ as $n \rightarrow \infty$,

- $\hat{\gamma}_{n}(x)$ such that $\sigma_{n}^{-1}\left(\hat{\gamma}_{n}(x)-\gamma(x)\right) \stackrel{d}{\longrightarrow} \mathcal{N}\left(0, v^{2}(x)\right)$ where $v^{2}(x)>0$.

Then, for all $x \in \mathbb{R}^{p}$,

$$
\frac{\sigma_{n}^{-1}}{\log \left(\alpha_{n} / \beta_{n}\right)}\left(\frac{\hat{q}_{n}^{W}\left(\beta_{n} \mid x\right)}{q\left(\beta_{n} \mid x\right)}-1\right) \stackrel{d}{\longrightarrow} \mathcal{N}\left(0, v^{2}(x)\right) .
$$

Note that, when $K$ is the pdf of the uniform distribution, this result is consistent with [21], Theorem 3, obtained in a fixed-design and functional setting.

\section{Numerical experiments on simulated data}

Here, we limit ourselves to one-dimensional random variables $X(p=1)$ uniformly distributed on $E=[0,1]$. Besides, $Y$ given $X=x$ is Fréchet distributed, its csf is given by $\bar{F}(y \mid x)=$ $\exp \left(-y^{-1 / \gamma(x)}\right)$, and the following conditional tail-index has been chosen:

$$
\gamma(x)=\frac{1}{2}\left(\frac{1}{10}+\sin (\pi x)\right)\left(\frac{11}{10}-\frac{1}{2} \exp \left(-64(x-1 / 2)^{2}\right)\right) .
$$

We focus on the estimation of conditional extreme quantiles $q\left(\alpha_{n} \mid x\right)=\left(-\log \alpha_{n}\right)^{-\gamma(x)}$ of order $\alpha_{n}=5 \log (n) / n$ which is inspired from the lower bound given in Remark 1 . To this end, we use the estimator introduced in (2) with a bi-quadratic kernel defined as $K(x)=\frac{15}{16}\left(1-x^{2}\right)^{2} \mathbb{I}\{|x| \leq 1\}$. The choice of the bandwidth $h$ is an important issue. In the following, we propose a data-driven strategy to select its value in a set $\mathcal{H}=\left\{h_{1} \leq h_{2} \leq \cdots \leq h_{M}\right\}$ where $h_{1}=1 /(5 \log n)$ and $h_{M}=1 / 2$. The minimum value $h_{1}$ is chosen to obtain approximately $2 n h_{1} \alpha_{n}=2$ observations in the area $\left[x-h_{1}, x+h_{1}\right] \times\left[q\left(\alpha_{n} \mid x\right), \infty\right)$ while the maximum value $h_{M}$ corresponds to a smoothing on the whole $[0,1]$ interval. The points $h_{2}, \ldots, h_{M-1}$ are regularly distributed in $\left[h_{1}, h_{M}\right]$ and $M=50$ is used in practice. Two strategies are compared. The first one is derived from the cross-validation approach introduced in [43] and implemented for instance in [17]:

$$
h_{c v}=\arg \min _{h \in \mathcal{H}} \sum_{i=1}^{n} \sum_{j=1}^{n}\left(\mathbb{I}\left\{Y_{i} \geq Y_{j}\right\}-\hat{\bar{F}}_{n,-i}\left(Y_{j} \mid X_{i}\right)\right)^{2} \text {, }
$$

where $\hat{\bar{F}}_{n,-i}$ is the estimator (depending on $h$ ) given in (1) computed from the sample $\left\{\left(X_{\ell}, Y_{\ell}\right), 1 \leq\right.$ $\ell \leq n, \ell \neq i\}$. The second approach is the oracle strategy which consists in minimizing a distance $\Delta$ between the estimated conditional extreme quantile and the true one:

$$
h_{\text {oracle }}=\arg \min _{h \in \mathcal{H}} \Delta\left(q\left(\alpha_{n} \mid .\right), \hat{q}_{n}\left(\alpha_{n} \mid .\right)\right) \text {, }
$$


with

$$
\Delta\left(q\left(\alpha_{n} \mid .\right), \hat{q}_{n}\left(\alpha_{n} \mid .\right)\right)=\left\{\frac{1}{L} \sum_{\ell=1}^{L}\left(\hat{q}_{n}\left(\alpha_{n} \mid t_{\ell}\right)-q\left(\alpha_{n} \mid t_{\ell}\right)\right)^{2}\right\}^{1 / 2}
$$

and where $t_{1}, \ldots, t_{L}$ are regularly distributed on $[0,1]$. Of course, this method cannot be applied in practical situations where $q\left(\alpha_{n} \mid\right.$.) is unknown. However, it provides us the lower bound on the distance $\Delta$ that can be reached with our estimator.

The finite sample performance of these strategies is assessed on $N=100$ replications of the samples $\left\{\left(X_{i}, Y_{i}\right), i=1, \ldots, n\right\}$ of size $n \in\{300,1000\}$. The bandwidths $h_{c v}^{(r)}$ and $h_{\text {oracle }}^{(r)}$ are computed on each replication $r \in\{1, \ldots, N\}$ with the two strategies and the corresponding errors (7) are denoted by $\Delta_{c v}^{(r)}$ and $\Delta_{\text {oracle }}^{(r)}$. As an illustration, the empirical distributions of $\Delta_{c v}^{(r)}$ and $\Delta_{\text {oracle }}^{(r)}$, obtained on samples of size $n=300$, are superimposed on Figure 1. It appears that the mean errors are approximately equal. Let us also remark that the cross-validation errors seem to have a heavier right-tail than the oracle errors. Similar conclusions have been drawn for $n=1000$. Let us now focus on the estimators $\hat{q}_{n}\left(\alpha_{n} \mid\right.$.) computed from the bandwidths associated to the quantiles of order $10 \%, 50 \%$ and $90 \%$ of the cross-validation error distribution. They respectively correspond to the best $10 \%$ estimator (in terms of the $\Delta$ error (7)), median estimator and worst $10 \%$ estimator obtained with the cross-validation criterion. In Figure $2(n=1000)$ they are superimposed to the true conditional quantile as well as to the estimated one with the oracle strategy on the same replication. The vertical axis is represented in a logarithmic scale for the visualization sake. One can see that the best $10 \%$ and median estimators obtained with the cross-validation strategy are quite stable and as good as the ones obtained by the oracle strategy. In contrast, the worst $10 \%$ estimator obtained with the cross-validation strategy is less accurate than the corresponding oracle estimator, the reason being that the cross-validation strategy is more sensitive to the extreme points than the oracle one. This phenomena may explain the heavy right-tail of the cross-validation error observed on Figure 1. However, the cross-validation criterion provides satisfactory results in most of the cases. Let us observe that the results obtained with $n=300$ (not displayed here for the sake of conciseness) are qualitatively equivalent. This phenomena is a consequence of $\alpha_{1000}<\alpha_{300}$ which means that estimating $q\left(\alpha_{1000} \mid\right.$.) is more difficult than estimating $q\left(\alpha_{300} \mid\right.$.). Finally, the tail index estimated by $\hat{\gamma}_{n}^{\mathrm{H}}(x)$ is compared to the true one $\gamma(x)$ on Figure 3 for different values of the $\alpha_{n}$ sequence. The automatic choice of $\alpha_{n}$, which is a recurrent problem in extreme-value theory, is not addressed here. We refer to [19] for an heuristical solution.

\section{Illustration on real data}

As an illustration, we propose an application of our methodology in a hyper-spectral remote sensing framework. The original data consists of $n=3184$ pairs denoted by $\left(S_{i}, P_{i}\right), i=1, \ldots, n$. Each $S_{i}$ is a spectra (in some high-dimensional space $E$ ) representing the intensity of light energy 
reflected from materials on Mars as it varies across different wavelengths. The analysis of these spectral signatures allows the identification of the physical, chemical or mineralogical properties of the surface that may help to understand the geological history of planets. We refer to [5] for a detailed presentation of the physical context. Here, we focus on the physical parameter $P_{i} \in[0,1]$ representing a $\mathrm{CO}_{2}$ proportion. Dimension reduction techniques [6] have shown that a one-dimensional predictor $X_{i}=<b, S_{i}>$ is sufficient to predict $P_{i}$, with $b \in E$ and where $<., .>$ denotes a dot-product in $E$ that we do not specify here. Finally, the variables of interest are $Y_{i}=\left(1-P_{i}\right)^{-1}-\left(1-P_{\min }\right)^{-1}$ where $P_{\min }$ is chosen such that $Y_{i} \in[0, \infty)$. The resulting scatterplot is depicted on Figure 6. We focus on the estimation of conditional extreme quantiles of order $\alpha_{n}=\zeta \log (n) / n$ with $\zeta \in\{5,10,20\}$. The above described procedure yields $h_{c v} \simeq 0.12$. Let us denote by $Z_{j}, j=1, \ldots, m(x)$ the observations $Y_{i}$ such that $X_{i} \in\left[x-h_{c v}, x+h_{c v}\right], i=1, \ldots, n$. Our approach relies on the property that the $Z_{j}, j=1, \ldots, m(x)$ are approximately distributed from (F.1). This assumption can be graphically checked on the QQ-plots obtained by drawing $k$ log-spacings versus standard exponential quantiles:

$$
\left(\log \frac{k}{j}, \log \frac{Z_{m(x)-j+1, m(x)}}{Z_{m(x)-k, m(x)}}, j=1, \ldots, k\right) .
$$

These QQ-plots are based on the property that, under (F.1), the $k \log$-spacings $\log \left(Z_{m(x)-j+1, m(x)}\right)-$ $\log \left(Z_{m(x)-k+1, m(x)}\right)$ are approximately distributed from an exponential distribution with scale parameter $\gamma(x)$, see [14], Section 6.2, for a review on exploratory data analysis methods for extremes. The obtained QQ-plots at three different locations $(x=0.25, x=0.50$ and $x=0.75)$ are presented on Figure 4 with $k=150$. Let us note that the plots are approximately linear, confirming the adequacy of the heavy-tailed model (F.1) to the dataset. The different slopes indicate some heterogeneity of the sample in terms of tail behavior. Figure 5 shows the obtained Hill-plots at three different locations i.e. $\hat{\gamma}_{n}^{\mathrm{H}}(x)$ for $x=0.25, x=0.50$ and $x=0.75$ (with $\tau_{j}=1 / j$ for $j=1, \ldots, 9$ ) as a function of the sample fraction $k$. Similarly to the unconditional case, the estimated conditional tail index depends on $k$ whose choice is difficult in practice. Here, $k=150$ could be a good choice in view of the QQ-plots (Figure 4) and because of the relative stability of the conditional Hill estimator (Figure 5) on its neighborhood. Finally, the estimated extreme level curves are superimposed to the scatter-plot on Figure 6.

\section{Appendix: Proofs}

\subsection{Preliminary results}

The first lemma is dedicated to the control of the local variations of the csf with respect to the covariate $x$ on a neighborhood of size $h$ when the quantity of interest $y$ goes to infinity. 
Lemma 1 Suppose (F.1), (L.1) and (L.2) hold. If $y_{n} \rightarrow \infty$ and $h \log y_{n} \rightarrow 0$ as $n \rightarrow \infty$, then

$$
\sup _{d\left(x, x^{\prime}\right) \leq h}\left|\frac{\bar{F}\left(y_{n} \mid x\right)}{\bar{F}\left(y_{n} \mid x^{\prime}\right)}-1\right|=O\left(h \log y_{n}\right) .
$$

Proof. Assumption (F.1) yields

$$
\begin{aligned}
\left|\log \left(\frac{\bar{F}\left(y_{n} \mid x\right)}{\bar{F}\left(y_{n} \mid x^{\prime}\right)}\right)\right| & \leq\left|\log y_{n}\right|\left(\left|\frac{1}{\gamma(x)}-\frac{1}{\gamma\left(x^{\prime}\right)}\right|+\left|\frac{\log \ell\left(y_{n} \mid x\right)}{\log y_{n}}-\frac{\log \ell\left(y_{n} \mid x^{\prime}\right)}{\log y_{n}}\right|\right) \\
& \leq\left(c_{\gamma}+c_{\ell}\right) \log y_{n} d\left(x, x^{\prime}\right),
\end{aligned}
$$

from (L.1), (L.2) and since, for $n$ large enough, $y_{n}>1$. Thus,

$$
\sup _{d\left(x, x^{\prime}\right) \leq h}\left|\log \left(\frac{\bar{F}\left(y_{n} \mid x\right)}{\bar{F}\left(y_{n} \mid x^{\prime}\right)}\right)\right|=O\left(h \log y_{n}\right) \rightarrow 0
$$

as $n \rightarrow \infty$ and taking account of $\log (u+1) \sim u$ as $u \rightarrow 0$ gives the result.

The second lemma is also of analytical nature. It provides a second order asymptotic expansion of the quantile function.

Lemma 2 Suppose (F.1), (F.2) and (F.3) hold.

(i) Let $0<\beta_{n}<\alpha_{n}$ with $\alpha_{n} \rightarrow 0$ as $n \rightarrow \infty$. Then,

$$
\left|\log q\left(\beta_{n} \mid x\right)-\log q\left(\alpha_{n} \mid x\right)+\gamma(x) \log \left(\beta_{n} / \alpha_{n}\right)\right|=O\left(\log \left(\alpha_{n} / \beta_{n}\right) \varepsilon\left(q\left(\alpha_{n} \mid x\right) \mid x\right)\right) .
$$

(ii) If, moreover, $\liminf \beta_{n} / \alpha_{n}>0$, then

$$
\frac{\beta_{n}^{\gamma(x)} q\left(\beta_{n} \mid x\right)}{\alpha_{n}^{\gamma(x)} q\left(\alpha_{n} \mid x\right)}=1+O\left(\varepsilon\left(q\left(\alpha_{n} \mid x\right) \mid x\right)\right) .
$$

Proof. (i) Let us introduce $\varphi(., x)=\log q(\exp () \mid x$.$) . We thus have$

$$
\begin{aligned}
\Delta_{n} & :=\log q\left(\beta_{n} \mid x\right)-\log q\left(\alpha_{n} \mid x\right)+\gamma(x) \log \left(\beta_{n} / \alpha_{n}\right) \\
& =\varphi\left(\log \beta_{n}, x\right)-\varphi\left(\log \alpha_{n}, x\right)+\gamma(x) \log \left(\beta_{n} / \alpha_{n}\right) .
\end{aligned}
$$

Under (F.1) and (F.2), Karamata representation (4) holds and thus $\varphi(., x)$ is differentiable. A first order expansion shows that there exists $\theta_{n} \in\left(\beta_{n}, \alpha_{n}\right)$ such that

$$
\begin{aligned}
\Delta_{n} & =\left(\gamma(x)+\varphi^{\prime}\left(\log \theta_{n}, x\right)\right) \log \left(\beta_{n} / \alpha_{n}\right) \\
& =\left(\gamma(x)+\frac{\bar{F}\left(q\left(\theta_{n} \mid x\right) \mid x\right)}{\bar{F}^{\prime}\left(q\left(\theta_{n} \mid x\right) \mid x\right) q\left(\theta_{n} \mid x\right)}\right) \log \left(\beta_{n} / \alpha_{n}\right) \\
& =\left(1+\left(\frac{\gamma(x) \ell^{\prime}\left(q\left(\theta_{n} \mid x\right) \mid x\right) q\left(\theta_{n} \mid x\right)}{\ell\left(q\left(\theta_{n} \mid x\right) \mid x\right)}-1\right)^{-1}\right) \gamma(x) \log \left(\beta_{n} / \alpha_{n}\right) \\
& =\left(1+\frac{1}{\gamma(x) \varepsilon\left(q\left(\theta_{n} \mid x\right) \mid x\right)-1}\right) \gamma(x) \log \left(\beta_{n} / \alpha_{n}\right) \\
& =-\gamma^{2}(x) \varepsilon\left(q\left(\theta_{n} \mid x\right) \mid x\right) \log \left(\beta_{n} / \alpha_{n}\right)(1+o(1)) .
\end{aligned}
$$

Since $q(. \mid x)$ and $|\varepsilon(. \mid x)|$ are both ultimately non-increasing, it follows that $\left|\varepsilon\left(q\left(\theta_{n} \mid x\right) \mid x\right)\right| \leq\left|\varepsilon\left(q\left(\alpha_{n} \mid x\right) \mid x\right)\right|$ and thus $\left|\Delta_{n}\right|=O\left(\log \left(\alpha_{n} / \beta_{n}\right) \varepsilon\left(q\left(\alpha_{n} \mid x\right) \mid x\right)\right)$, and the first part is proved.

(ii) Here, $0<\liminf \beta_{n} / \alpha_{n} \leq \limsup \beta_{n} / \alpha_{n} \leq 1$, and (i) entails $\left|\Delta_{n}\right|=O\left(\varepsilon\left(q\left(\alpha_{n} \mid x\right) \mid x\right)\right) \rightarrow 0$ as $n \rightarrow \infty$. As a consequence, $\exp \left(\Delta_{n}\right)=1+\Delta_{n}(1+o(1))$ and the conclusion follows. 
The following lemma provides a geometrical interpretation of the condition $n h^{p} \bar{F}\left(y_{n} \mid x\right) \rightarrow \infty$.

Lemma 3 Suppose (F.1), (L.1), (L.2), (L.3) hold and let $y_{n} \rightarrow \infty$ such that $h \log y_{n} \rightarrow 0$ as $n \rightarrow \infty$. Consider the region of $\mathbb{R}^{p+1}$ defined as $R_{n}(x)=B(x, h) \times\left(y_{n}, \infty\right)$ where $x \in \mathbb{R}^{p}$ is such that $g(x)>0$. Then, $\mathbb{P}\left(\exists i \in\{1, \ldots, n\},\left(X_{i}, Y_{i}\right) \in R_{n}(x)\right) \rightarrow 1$ as $n \rightarrow \infty$ if, and only if, $n h^{p} \bar{F}\left(y_{n} \mid x\right) \rightarrow \infty$.

Proof. Standard considerations lead to

$$
\mathbb{P}\left(\exists i \in\{1, \ldots, n\},\left(X_{i}, Y_{i}\right) \in R_{n}(x)\right)=1-\left(1-\mathbb{P}\left(\left(X_{1}, Y_{1}\right) \in R_{n}(x)\right)\right)^{n},
$$

and, in view of (L.3) and Lemma 1,

$$
\begin{aligned}
\left.\mathbb{P}\left(\left(X_{1}, Y_{1}\right) \in R_{n}(x)\right)\right) & =\int_{B(x, h)} \bar{F}\left(y_{n} \mid u\right) g(u) d u=\bar{F}\left(y_{n} \mid x\right) g(x)\left(1+O\left(h \log y_{n}\right)\right) \int_{B(x, h)} d u \\
& =v_{p} h^{p} \bar{F}\left(y_{n} \mid x\right) g(x)\left(1+O\left(h \log y_{n}\right)\right),
\end{aligned}
$$

where $v_{p}$ is the volume of the unit ball in $\mathbb{R}^{p}$. Clearly, this probability converges to 0 as $n \rightarrow \infty$ and thus (8) can be rewritten as

$$
\mathbb{P}\left(\exists i \in\{1, \ldots, n\},\left(X_{i}, Y_{i}\right) \in R_{n}(x)\right)=1-\exp \left(-v_{p} g(x) n h^{p} \bar{F}\left(y_{n} \mid x\right)(1+o(1))\right),
$$

which converges to 1 if and only if $n h^{p} \bar{F}\left(y_{n} \mid x\right) \rightarrow \infty$.

Let us remark that the kernel estimator (1) can be rewritten as $\hat{\bar{F}}_{n}(y \mid x)=\hat{\psi}_{n}(y, x) / \hat{g}_{n}(x)$ where

$$
\hat{\psi}_{n}(y, x)=\frac{1}{n} \sum_{i=1}^{n} K_{h}\left(x-X_{i}\right) \mathbb{I}\left\{Y_{i}>y\right\}
$$

is an estimator of $\psi(y, x)=\bar{F}(y \mid x) g(x)$ and $\hat{g}_{n}(x)$ is the classical kernel estimator (6) of the pdf $g(x)$. Lemma 4 gives standard results on the kernel estimator (see [9], Proposition 2.1 and Proposition 2.2 for a proof) whereas Lemma 5 is dedicated to the asymptotic properties of $\hat{\psi}_{n}(y, x)$.

Lemma 4 Suppose (L.3), (K) hold. If $n h^{p} \rightarrow \infty$, then, for all $x \in \mathbb{R}^{p}$,

(i) $\mathbb{E}\left(\hat{g}_{n}(x)-g(x)\right)=O(h)$,

(ii) $\operatorname{var}\left(\hat{g}_{n}(x)\right)=\frac{g(x)\|K\|_{2}^{2}}{n h^{p}}(1+o(1))$.

Therefore, under the assumptions of the above lemma, $\hat{g}_{n}(x)$ converges to $g(x)$ in probability.

Lemma 5 Suppose (F.1), (L.1), (L.2), (L.3) and (K) hold. Let us introduce

- $0<a_{1}<a_{2}<\cdots<a_{J}$ where $J$ is a positive integer,

- $y_{n} \rightarrow \infty$ such that $h \log y_{n} \rightarrow 0$ and $n h^{p} \bar{F}\left(y_{n} \mid x\right) \rightarrow \infty$ as $n \rightarrow \infty$,

- $y_{n, j}=a_{j} y_{n}$ for $j=1, \ldots, J$. 
Then, for all $x \in \mathbb{R}^{p}$ such that $g(x)>0$,

(i) $\mathbb{E}\left(\hat{\psi}_{n}\left(y_{n, j}, x\right)\right)=\psi\left(y_{n, j}, x\right)\left(1+O\left(h \log y_{n}\right)\right)$, for $j=1, \ldots, J$.

(ii) The random vector

$$
\left\{\sqrt{n h^{p} \psi\left(y_{n}, x\right)}\left(\frac{\hat{\psi}_{n}\left(y_{n, j}, x\right)-\mathbb{E}\left(\hat{\psi}_{n}\left(y_{n, j}, x\right)\right)}{\psi\left(y_{n, j}, x\right)}\right)\right\}_{j=1, \ldots, J}
$$

is asymptotically Gaussian, centered, with covariance matrix $\|K\|_{2}^{2} C(x)$ where $C_{j, j^{\prime}}(x)=$ $a_{j \wedge j^{\prime}}^{1 / \gamma(x)}$ for $\left(j, j^{\prime}\right) \in\{1, \ldots, J\}^{2}$.

Proof. (i) Since the $\left(X_{i}, Y_{i}\right), i=1, \ldots, n$ are identically distributed, we have

$$
\mathbb{E}\left(\hat{\psi}_{n}\left(y_{n, j}, x\right)\right)=\int_{\mathbb{R}^{p}} K_{h}(x-t) \bar{F}\left(y_{n, j} \mid t\right) g(t) d t=\int_{S} K(u) \bar{F}\left(y_{n, j} \mid x-h u\right) g(x-h u) d u,
$$

under (K). Let us now consider

$$
\begin{aligned}
\left|\mathbb{E}\left(\hat{\psi}_{n}\left(y_{n, j}, x\right)\right)-\psi\left(y_{n, j}, x\right)\right| & \leq \bar{F}\left(y_{n, j} \mid x\right) \int_{S} K(u)|g(x-h u)-g(x)| d u \\
& +\bar{F}\left(y_{n, j} \mid x\right) \int_{S} K(u)\left|\frac{\bar{F}\left(y_{n, j} \mid x-h u\right)}{\bar{F}\left(y_{n, j} \mid x\right)}-1\right| g(x-h u) d u .
\end{aligned}
$$

Under (L.3), and since $g(x)>0$, we have

$$
(9) \leq \bar{F}\left(y_{n, j} \mid x\right) c_{g} h \int_{S} d(u, 0) K(u) d u=\psi\left(y_{n, j}, x\right) O(h) .
$$

Besides, Lemma 1 implies that

$$
\sup _{u \in S}\left|\frac{\bar{F}\left(y_{n, j} \mid x-h u\right)}{\bar{F}\left(y_{n, j} \mid x\right)}-1\right|=O\left(h \log y_{n, j}\right)=O\left(h \log y_{n}\right)
$$

and therefore, in view of (11),

$$
\begin{aligned}
(10) & =\bar{F}\left(y_{n, j} \mid x\right) O\left(h \log y_{n}\right) \int_{S} K(u) g(x-h u) d u=\bar{F}\left(y_{n, j} \mid x\right) g(x) O\left(h \log y_{n}\right)(1+o(1)) \\
& =\psi\left(y_{n, j}, x\right) O\left(h \log y_{n}\right) .
\end{aligned}
$$

Combining (11) and (12) concludes the first part of the proof.

(ii) Let $\beta \neq 0$ in $\mathbb{R}^{J}, \Lambda_{n}(x)=\left(n h^{p} \psi\left(y_{n}, x\right)\right)^{-1 / 2}$, and consider the random variable

$$
\begin{aligned}
\Psi_{n} & =\sum_{j=1}^{J} \beta_{j}\left(\frac{\hat{\psi}_{n}\left(y_{n, j}, x\right)-\mathbb{E}\left(\hat{\psi}_{n}\left(y_{n, j}, x\right)\right)}{\Lambda_{n}(x) \psi\left(y_{n, j}, x\right)}\right) \\
& =\sum_{i=1}^{n} \frac{1}{n \Lambda_{n}(x)}\left\{\sum_{j=1}^{J} \frac{\beta_{j} K_{h}\left(x-X_{i}\right) \mathbb{I}\left\{Y_{i} \geq y_{n, j}\right\}}{\psi\left(y_{n, j}, x\right)}-\mathbb{E}\left(\sum_{j=1}^{J} \frac{\beta_{j} K_{h}\left(x-X_{i}\right) \mathbb{I}\left\{Y_{i} \geq y_{n, j}\right\}}{\psi\left(y_{n, j}, x\right)}\right)\right\} \\
& =: \sum_{i=1}^{n} Z_{i, n} .
\end{aligned}
$$


Clearly, $\left\{Z_{i, n}, i=1, \ldots, n\right\}$ is a set of centered, independent and identically distributed random variables with variance

$$
\operatorname{var}\left(Z_{i, n}\right)=\frac{1}{n^{2} h^{2 p} \Lambda_{n}^{2}(x)} \operatorname{var}\left(\sum_{j=1}^{J} \beta_{j} K\left(\frac{x-X_{i}}{h}\right) \frac{\mathbb{I}\left\{Y_{i} \geq y_{n, j}\right\}}{\psi\left(y_{n, j}, x\right)}\right)=\frac{1}{n^{2} h^{p} \Lambda_{n}^{2}(x)} \beta^{t} B \beta,
$$

where $B$ is the $J \times J$ covariance matrix with coefficients defined for $\left(j, j^{\prime}\right) \in\{1, \ldots, J\}^{2}$ by

$$
\begin{aligned}
B_{j, j^{\prime}} & =\frac{A_{j, j^{\prime}}}{\psi\left(y_{n, j}, x\right) \psi\left(y_{n, j^{\prime}}, x\right)}, \\
A_{j, j^{\prime}} & =\frac{1}{h^{p}} \operatorname{cov}\left(K\left(\frac{x-X}{h}\right) \mathbb{I}\left\{Y \geq y_{n, j}\right\}, K\left(\frac{x-X}{h}\right) \mathbb{I}\left\{Y \geq y_{n, j^{\prime}}\right\}\right) \\
& =\|K\|_{2}^{2} \mathbb{E}\left(\frac{1}{h^{p}} Q\left(\frac{x-X}{h}\right) \mathbb{I}\left\{Y \geq y_{n, j} \vee y_{n, j^{\prime}}\right\}\right) \\
& -h^{p} \mathbb{E}\left(K_{h}(x-X) \mathbb{I}\left\{Y \geq y_{n, j}\right\}\right) \mathbb{E}\left(K_{h}(x-X) \mathbb{I}\left\{Y \geq y_{n, j^{\prime}}\right\}\right),
\end{aligned}
$$

with $Q()=.: K^{2}(.) /\|K\|_{2}^{2}$ also satisfying assumption (K). As a consequence, the three above expectations are of the same nature. Thus, remarking that, for $n$ large enough, $y_{n, j} \vee y_{n, j^{\prime}}=y_{n, j \vee j^{\prime}}$, part (i) of the proof implies

$$
A_{j, j^{\prime}}=\|K\|_{2}^{2} \psi\left(y_{n, j \vee j^{\prime}}, x\right)\left(1+O\left(h \log y_{n}\right)\right)-h^{p} \psi\left(y_{n, j}, x\right) \psi\left(y_{n, j^{\prime}}, x\right)\left(1+O\left(h \log y_{n}\right)\right)
$$

leading to

$$
B_{j, j^{\prime}}=\frac{\|K\|_{2}^{2}}{\psi\left(y_{n, j \wedge j^{\prime}}, x\right)}\left(1+O\left(h \log y_{n}\right)\right)-h^{p}\left(1+O\left(h \log y_{n}\right)\right)=\frac{\|K\|_{2}^{2}}{\psi\left(y_{n, j \wedge j^{\prime}}, x\right)}(1+o(1)),
$$

since $\psi\left(y_{n, j \wedge j^{\prime}}, x\right) \rightarrow 0$ as $n \rightarrow \infty$. Now, from the regular variation property (F.1), it is easily seen that $\psi\left(y_{n, j \wedge j^{\prime}}, x\right)=a_{j \wedge j^{\prime}}^{-1 / \gamma(x)} \psi\left(y_{n}, x\right)(1+o(1))$ entailing $B_{j, j^{\prime}}=\frac{\|K\|_{2}^{2} C_{j, j^{\prime}}(x)}{\psi\left(y_{n}, x\right)}(1+o(1))$ and therefore, $\operatorname{var}\left(Z_{i, n}\right) \sim\|K\|_{2}^{2} \beta^{t} C(x) \beta / n$, for all $i=1, \ldots, n$. As a preliminary conclusion, the variance of $\Psi_{n}$ converges to $\|K\|_{2}^{2} \beta^{t} C(x) \beta$. Consequently, Lyapounov criteria for the asymptotic normality of sums of triangular arrays reduces to $\sum_{i=1}^{n} \mathbb{E}\left|Z_{i, n}\right|^{3}=n \mathbb{E}\left|Z_{1, n}\right|^{3} \rightarrow 0$. Remark that $Z_{1, n}$ is a bounded random variable:

$$
\left|Z_{1, n}\right| \leq \frac{2\|K\|_{\infty} \sum_{j=1}^{J}\left|\beta_{j}\right|}{n \Lambda_{n}(x) h^{p} \psi\left(y_{n, J}, x\right)}=2\|K\|_{\infty} a_{J}^{1 / \gamma(x)} \sum_{j=1}^{J}\left|\beta_{j}\right| \Lambda_{n}(x)(1+o(1))
$$

and thus,

$$
\begin{aligned}
n \mathbb{E}\left|Z_{1, n}\right|^{3} & \leq 2\|K\|_{\infty} a_{J}^{1 / \gamma(x)} \sum_{j=1}^{J}\left|\beta_{j}\right| \Lambda_{n}(x) n \operatorname{var}\left(Z_{1, n}\right)(1+o(1)) \\
& =2\|K\|_{\infty}\|K\|_{2}^{2} a_{J}^{1 / \gamma(x)} \sum_{j=1}^{J}\left|\beta_{j}\right| \beta^{t} C(x) \beta \Lambda_{n}(x)(1+o(1)) \rightarrow 0
\end{aligned}
$$

as $n \rightarrow \infty$. As a conclusion, $\Psi_{n}$ converges in distribution to a centered Gaussian random variable with variance $\|K\|_{2}^{2} \beta^{t} C(x) \beta$ for all $\beta \neq 0$ in $\mathbb{R}^{p}$. The result is proved. 


\subsection{Proofs of main results}

Proof of Theorem 1. Keeping in mind the notations of Lemma 5, the following expansion holds

$$
\Lambda_{n}^{-1}(x) \sum_{j=1}^{J} \beta_{j}\left(\frac{\hat{\bar{F}}_{n}\left(y_{n, j} \mid x\right)}{\bar{F}\left(y_{n, j} \mid x\right)}-1\right)=\frac{\Delta_{1, n}+\Delta_{2, n}-\Delta_{3, n}}{\hat{g}_{n}(x)},
$$

where

$$
\begin{aligned}
& \Delta_{1, n}=g(x) \Lambda_{n}^{-1}(x) \sum_{j=1}^{J} \beta_{j}\left(\frac{\hat{\psi}_{n}\left(y_{n, j}, x\right)-\mathbb{E}\left(\hat{\psi}_{n}\left(y_{n, j}, x\right)\right)}{\psi\left(y_{n, j}, x\right)}\right) \\
& \Delta_{2, n}=g(x) \Lambda_{n}^{-1}(x) \sum_{j=1}^{J} \beta_{j}\left(\frac{\mathbb{E}\left(\hat{\psi}_{n}\left(y_{n, j}, x\right)\right)-\psi\left(y_{n, j}, x\right)}{\psi\left(y_{n, j}, x\right)}\right) \\
& \Delta_{3, n}=\left(\sum_{j=1}^{J} \beta_{j}\right) \Lambda_{n}^{-1}(x)\left(\hat{g}_{n}(x)-g(x)\right) .
\end{aligned}
$$

Let us highlight that assumptions $n h^{p+2} \log ^{2}\left(y_{n}\right) \bar{F}\left(y_{n} \mid x\right) \rightarrow 0$ and $n h^{p} \bar{F}\left(y_{n} \mid x\right) \rightarrow \infty$ imply that $h \log y_{n} \rightarrow 0$ as $n \rightarrow \infty$. Thus, from Lemma 5 (ii), the random term $\Delta_{1, n}$ can be rewritten as

$$
\Delta_{1, n}=g(x)\|K\|_{2} \sqrt{\beta^{t} C(x) \beta} \xi_{n},
$$

where $\xi_{n}$ converges to a standard Gaussian random variable. The nonrandom term $\Delta_{2, n}$ is controlled with Lemma 5(i):

$$
\Delta_{2, n}=O\left(\Lambda_{n}^{-1}(x) h \log y_{n}\right)=O\left(n h^{p+2} \bar{F}\left(y_{n} \mid x\right) \log ^{2}\left(y_{n}\right)\right)^{1 / 2}=o(1) .
$$

Finally, $\Delta_{3, n}$ is a classical term in kernel density estimation, which can be bounded by Lemma 4 :

$$
\Delta_{3, n}=O\left(h \Lambda_{n}^{-1}(x)\right)+O_{P}\left(\Lambda_{n}^{-1}(x)\left(n h^{p}\right)^{-1 / 2}\right)=O\left(n h^{p+2} \bar{F}\left(y_{n} \mid x\right)\right)^{1 / 2}+O_{P}\left(\bar{F}\left(y_{n} \mid x\right)\right)^{1 / 2}=o_{P}(1) .
$$

Collecting (13)-(16), it follows that

$$
\hat{g}_{n}(x) \Lambda_{n}^{-1}(x) \sum_{j=1}^{J} \beta_{j}\left(\frac{\hat{\bar{F}}_{n}\left(y_{n, j} \mid x\right)}{\bar{F}\left(y_{n, j} \mid x\right)}-1\right)=g(x)\|K\|_{2} \sqrt{\beta^{t} C(x) \beta} \xi_{n}+o_{P}(1) .
$$

Finally, $\hat{g}_{n}(x) \stackrel{P}{\longrightarrow} g(x)$ yields

$$
\sqrt{n h^{p} \bar{F}\left(y_{n} \mid x\right)} \sum_{j=1}^{J} \beta_{j}\left(\frac{\hat{\bar{F}}_{n}\left(y_{n, j} \mid x\right)}{\bar{F}\left(y_{n, j} \mid x\right)}-1\right)=\|K\|_{2} \sqrt{\frac{\beta^{t} C(x) \beta}{g(x)}} \xi_{n}+o_{P}(1)
$$

and the result is proved.

Proof of Theorem 2. Introduce for $j=1, \ldots, J$,

$$
\begin{aligned}
\sigma_{n, j}(x) & =q\left(\alpha_{n, j} \mid x\right)\left(n h^{p} \alpha_{n}\right)^{-1 / 2} \\
v_{n, j}(x) & =\alpha_{n, j}^{-1} \gamma(x)\left(n h^{p} \alpha_{n}\right)^{1 / 2} \\
W_{n, j}(x) & =v_{n, j}(x)\left(\hat{\bar{F}}_{n}\left(q\left(\alpha_{n, j} \mid x\right)+\sigma_{n, j}(x) z_{j} \mid x\right)-\bar{F}\left(q\left(\alpha_{n, j} \mid x\right)+\sigma_{n, j}(x) z_{j} \mid x\right)\right) \\
a_{n, j}(x) & =v_{n, j}(x)\left(\alpha_{n, j}-\bar{F}\left(q\left(\alpha_{n, j} \mid x\right)+\sigma_{n, j}(x) z_{j} \mid x\right)\right)
\end{aligned}
$$


and $z_{j} \in \mathbb{R}$. We examine the asymptotic behavior of $J$-variate function defined by $\Phi_{n}\left(z_{1}, \ldots, z_{J}\right)=\mathbb{P}\left(\bigcap_{j=1}^{J}\left\{\sigma_{n, j}^{-1}(x)\left(\hat{q}_{n}\left(\alpha_{n, j} \mid x\right)-q\left(\alpha_{n, j} \mid x\right)\right) \leq z_{j}\right\}\right)=\mathbb{P}\left(\bigcap_{j=1}^{J}\left\{W_{n, j}(x) \leq a_{n, j}(x)\right\}\right)$.

Let us first focus on the nonrandom term $a_{n, j}(x)$. From assumptions (F.1) and (F.2), Karamata representation (4) shows that $\bar{F}(. \mid x)$ is differentiable. Thus, for each $j \in\{1, \ldots, J\}$ there exists $\theta_{n, j} \in(0,1)$ such that

$$
\bar{F}\left(q\left(\alpha_{n, j} \mid x\right) \mid x\right)-\bar{F}\left(q\left(\alpha_{n, j} \mid x\right)+\sigma_{n, j}(x) z_{j} \mid x\right)=-\sigma_{n, j}(x) z_{j} \bar{F}^{\prime}\left(q_{n, j} \mid x\right),
$$

where $q_{n, j}=q\left(\alpha_{n, j} \mid x\right)+\theta_{n, j} \sigma_{n, j}(x) z_{j}$. It is clear that $q\left(\alpha_{n, j} \mid x\right) \rightarrow \infty$ and $\sigma_{n, j}(x) / q\left(\alpha_{n, j} \mid x\right) \rightarrow 0$ as $n \rightarrow \infty$. As a consequence, $q_{n, j} \rightarrow \infty$ and thus Karamata representation (4) entails

$$
\lim _{n \rightarrow \infty} \frac{q_{n, j} \bar{F}^{\prime}\left(q_{n, j} \mid x\right)}{\bar{F}\left(q_{n, j} \mid x\right)}=-1 / \gamma(x) .
$$

Moreover, since $q_{n, j} \sim q\left(\alpha_{n, j} \mid x\right)$ as $n \rightarrow \infty$ and $\bar{F}(. \mid x)$ is regularly varying, it follows that $\bar{F}\left(q_{n, j} \mid x\right) \sim \bar{F}\left(q\left(\alpha_{n, j} \mid x\right) \mid x\right)=\alpha_{n, j}$. In view of (17) and (18), we end up with

$$
a_{n, j}(x)=\frac{v_{n, j}(x) \sigma_{n, j}(x) \alpha_{n, j} z_{j}}{\gamma(x) q\left(\alpha_{n, j} \mid x\right)}(1+o(1))=z_{j}(1+o(1)) .
$$

Let us now turn to the random term $W_{n, j}(x)$. Defining $a_{j}=\tau_{j}^{-\gamma(x)}, y_{n, j}=q\left(\alpha_{n, j} \mid x\right)+\sigma_{n, j}(x) z_{j}$ for $j=1, \ldots, J$ and $y_{n}=q\left(\alpha_{n} \mid x\right)$, we have $y_{n, j} \sim q\left(\alpha_{n, j} \mid x\right) \sim a_{j} y_{n}$ since $q(. \mid x)$ is regularly varying a 0 with index $-\gamma(x)$. Using the same argument, it is easily shown that $\log y_{n} \sim-\gamma(x) \log \alpha_{n}$. As a consequence, Theorem 1 applies and the random vector

$$
\left\{\frac{\sqrt{n h^{p} \bar{F}\left(y_{n} \mid x\right)}}{v_{n, j}(x) \bar{F}\left(y_{n, j} \mid x\right)} W_{n, j}\right\}_{j=1, \ldots, J}=(1+o(1))\left\{\frac{W_{n, j}}{\gamma(x)}\right\}_{j=1, \ldots, J}
$$

converges to a centered Gaussian random variable with covariance matrix $\frac{\|K\|_{2}^{2}}{g(x)} C(x)$. Taking account of (19), we obtain that $\Phi_{n}\left(z_{1}, \ldots, z_{J}\right)$ converges to the cumulative distribution function of a centered Gaussian distribution with covariance matrix $\frac{\|K\|_{2}^{2} \gamma^{2}(x)}{g(x)} C(x)$ evaluated at $\left(z_{1}, \ldots, z_{J}\right)$, which is the desired result.

Proof of Corollary 1. Introducing $\alpha_{n, j}=\tau_{j} \alpha_{n}$ for all $j \in\{1,2,3\}$ with $\tau_{1}=4, \tau_{2}=2$ and $\tau_{3}=1$, Theorem 2 shows that $\hat{q}_{n}\left(\alpha_{n, j} \mid x\right)=q\left(\alpha_{n, j} \mid x\right)\left(1+\sigma_{n} \xi_{n, j}\right)$ for $j \in\{1,2,3\}$ where $\left(\xi_{n, 1}, \xi_{n, 2}, \xi_{n, 3}\right)^{t}$ converges to a centered Gaussian random vector with covariance matrix

$$
\|K\|_{2}^{2} \gamma^{2}(x) / g(x)\left[\begin{array}{ccc}
1 / 4 & 1 / 4 & 1 / 4 \\
1 / 4 & 1 / 2 & 1 / 2 \\
1 / 4 & 1 / 2 & 1
\end{array}\right]
$$


Replacing in $\hat{\gamma}_{n}^{\mathrm{P}}(x)$ yields

$$
\begin{aligned}
(\log 2) \hat{\gamma}_{n}^{\mathrm{P}}(x) & =\log \left(\frac{q\left(\alpha_{n, 3} \mid x\right)}{q\left(\alpha_{n, 2} \mid x\right)}\left(1+\sigma_{n} \xi_{n, 3}\right)-1-\sigma_{n} \xi_{n, 2}\right) \\
& -\log \left(1+\sigma_{n} \xi_{n, 2}-\frac{q\left(\alpha_{n, 1} \mid x\right)}{q\left(\alpha_{n, 2} \mid x\right)}\left(1+\sigma_{n} \xi_{n, 1}\right)\right) \\
& =\log \left(2^{\gamma(x)}\left(1+O\left(\varepsilon\left(q\left(\alpha_{n, 2} \mid x\right) \mid x\right)\right)\right)\left(1+\sigma_{n} \xi_{n, 3}\right)-1-\sigma_{n} \xi_{n, 2}\right) \\
& -\log \left(1+\sigma_{n} \xi_{n, 2}-2^{-\gamma(x)}\left(1+O\left(\varepsilon\left(q\left(\alpha_{n, 2} \mid x\right) \mid x\right)\right)\right)\left(1+\sigma_{n} \xi_{n, 1}\right)\right)
\end{aligned}
$$

in view of Lemma 2(i). As a consequence of assumption $\left.\varepsilon\left(q\left(\alpha_{n, 2} \mid x\right) \mid x\right)\right) / \sigma_{n} \rightarrow 0$, we obtain

$$
\begin{aligned}
(\log 2) \hat{\gamma}_{n}^{\mathrm{P}}(x) & =\log \left(2^{\gamma(x)}-1+\sigma_{n}\left(2^{\gamma(x)} \xi_{n, 3}-\xi_{n, 2}+o_{P}(1)\right)\right) \\
& -\log \left(1-2^{-\gamma(x)}+\sigma_{n}\left(\xi_{n, 2}-2^{-\gamma(x)} \xi_{n, 1}+o_{P}(1)\right)\right) .
\end{aligned}
$$

Standard calculations lead to

$$
\sigma_{n}^{-1}(\log 2)\left(\hat{\gamma}_{n}^{\mathrm{P}}(x)-\gamma(x)\right)=\frac{\xi_{n, 1}-\left(1+2^{\gamma(x)}\right) \xi_{n, 2}+2^{\gamma(x)} \xi_{n, 3}}{2^{\gamma(x)}-1}+o_{P}(1),
$$

which converges to a centered Gaussian random variable with variance $\frac{\|K\|_{2}^{2} \gamma^{2}(x)\left(2^{2 \gamma(x)+1}+1\right)^{2}}{4\left(2^{\gamma(x)}-1\right)^{2} g(x)}$, and the conclusion follows.

Proof of Corollary 2. Left us define $c_{J}=\sum_{j=1}^{J} \log \left(1 / \tau_{j}\right), \alpha_{n, j}=\tau_{j} \alpha_{n}$ for $j=1, \ldots, J$ and consider the expansion $\sigma_{n}^{-1}\left(\hat{\gamma}_{n}^{\mathrm{H}}(x)-\gamma(x)\right)=T_{n, 1}+T_{n, 2}$ with

$$
\begin{aligned}
& T_{n, 1}=c_{J}^{-1} \sum_{j=1}^{J} \sigma_{n}^{-1}\left[\log \left(\frac{\hat{q}_{n}\left(\alpha_{n, j} \mid x\right)}{q\left(\alpha_{n, j} \mid x\right)}\right)-\log \left(\frac{\hat{q}_{n}\left(\alpha_{n, 1} \mid x\right)}{q\left(\alpha_{n, 1} \mid x\right)}\right)\right], \\
& T_{n, 2}=\sigma_{n}^{-1} c_{J}^{-1} \sum_{j=1}^{J} \log \left(\tau_{j}^{\gamma(x)} \frac{q\left(\alpha_{n, j} \mid x\right)}{q\left(\alpha_{n, 1} \mid x\right)}\right) .
\end{aligned}
$$

Let us first focus on $T_{n, 1}$. Following Theorem $2, \hat{q}_{n}\left(\alpha_{n, j} \mid x\right) / q\left(\alpha_{n, j} \mid x\right) \stackrel{P}{\longrightarrow} 1$ as $n \rightarrow \infty$ and thus

$$
\left\{\sigma_{n}^{-1} \log \left(\frac{\hat{q}_{n}\left(\alpha_{n, j} \mid x\right)}{q\left(\alpha_{n, j} \mid x\right)}\right)\right\}_{j=1, \ldots, J}=\left(1+o_{P}(1)\right)\left\{\sigma_{n}^{-1}\left(\frac{\hat{q}_{n}\left(\alpha_{n, j} \mid x\right)}{q\left(\alpha_{n, j} \mid x\right)}-1\right)\right\}_{j=1, \ldots, J}
$$

which converges in distribution to a centered Gaussian random vector with covariance matrix $\|K\|_{2}^{2} \gamma^{2}(x) \Sigma / g(x)$. As a consequence $T_{n, 1}$ converges in distribution to a centered Gaussian random variable with variance $\left(\beta^{t} \Sigma \beta\right)\|K\|_{2}^{2} \gamma^{2}(x) /\left(g(x) c_{J}^{2}\right)$ with $\beta=(1-J, 1, \ldots, 1)^{t} \in \mathbb{R}^{J}$. Straightforward calculations show that $\beta^{t} \Sigma \beta=V_{J} c_{J}^{2}$. Finally, $T_{n, 2}$ is a finite sum of $J$ terms which can be controlled with Lemma 2(ii): $T_{n, 2}=\sigma_{n}^{-1} O\left(\varepsilon\left(q\left(\alpha_{1, n} \mid x\right) \mid x\right)\right)$ with $\alpha_{1, n}=k / n$ and thus $T_{n, 2} \rightarrow 0$ as $\mathrm{n} \rightarrow \infty$ under the considered assumptions. 
Proof of Theorem 3. The proof is based on the following expansion:

$$
\frac{\sigma_{n}^{-1}}{\log \left(\alpha_{n} / \beta_{n}\right)}\left(\log \left(\hat{q}_{n}^{\mathrm{W}}\left(\beta_{n} \mid x\right)\right)-\log \left(q\left(\beta_{n} \mid x\right)\right)\right)=\frac{\sigma_{n}^{-1}}{\log \left(\alpha_{n} / \beta_{n}\right)}\left(Q_{n, 1}+Q_{n, 2}+Q_{n, 3}\right)
$$

where we have introduced

$$
\begin{aligned}
Q_{n, 1} & =\sigma_{n}^{-1}\left(\hat{\gamma}_{n}(x)-\gamma(x)\right) \\
Q_{n, 2} & =\frac{\sigma_{n}^{-1}}{\log \left(\alpha_{n} / \beta_{n}\right)} \log \left(\hat{q}_{n}\left(\alpha_{n} \mid x\right) / q\left(\alpha_{n} \mid x\right)\right), \\
Q_{n, 3} & =\frac{\sigma_{n}^{-1}}{\log \left(\alpha_{n} / \beta_{n}\right)}\left(\log q\left(\alpha_{n} \mid x\right)-\log q\left(\beta_{n} \mid x\right)+\gamma(x) \log \left(\alpha_{n} / \beta_{n}\right)\right) .
\end{aligned}
$$

First, $Q_{n, 1} \stackrel{d}{\longrightarrow} \mathcal{N}\left(0, v^{2}(x)\right)$ as a straightforward consequence of the assumptions. Second, Theorem 2 implies that $\hat{q}_{n}\left(\alpha_{n} \mid x\right) / q\left(\alpha_{n} \mid x\right) \stackrel{P}{\longrightarrow} 1$ and

$$
Q_{n, 2}=\frac{\sigma_{n}^{-1}}{\log \left(\alpha_{n} / \beta_{n}\right)}\left(\frac{\hat{q}_{n}\left(\alpha_{n} \mid x\right)}{q\left(\alpha_{n} \mid x\right)}-1\right)\left(1+o_{P}(1)\right)=\frac{O_{P}(1)}{\log \left(\alpha_{n} / \beta_{n}\right)} .
$$

Consequently, $Q_{n, 2} \stackrel{P}{\longrightarrow} 0$ as $n \rightarrow \infty$. Finally, from Lemma $2(\mathrm{i}), Q_{n, 3}=O\left(\sigma_{n}^{-1} \varepsilon\left(q\left(\alpha_{n} \mid x\right) \mid x\right)\right)$, which converges to 0 in view of the assumptions.

\section{Acknowledgments}

The authors are grateful to Sylvain Douté for his contribution to the hyper-spectral data and for fruitful discussions.

\section{References}

[1] M.I.F. Alves, M.I. Gomes, M.I. and L. de Haan. A new class of semi-parametric estimators of the second order parameter. Portugaliae Mathematica, 60:193-214, 2003.

[2] M.I.F. Alves, L. de Haan and T. Lin. Estimation of the parameter controlling the speed of convergence in extreme value theory, Mathematical Methods of Statistics, 12:155-176, 2003.

[3] J. Beirlant and Y. Goegebeur. Regression with response distributions of Pareto-type. Computational Statistics and Data Analysis, 42:595-619, 2003.

[4] A. Berlinet, A. Gannoun and E. Matzner-Løber. Asymptotic normality of convergent estimates of conditional quantiles. Statistics, 35:139-169, 2001.

[5] C. Bernard-Michel, S. Douté, M. Fauvel, L. Gardes and S. Girard. Retrieval of Mars surface physical properties from OMEGA hyperspectral images using Regularized Sliced Inverse Regression, Journal of Geophysical Research - Planets, 114, E06005, 2009. 
[6] C. Bernard-Michel, L. Gardes and S. Girard. Gaussian Regularized Sliced Inverse Regression, Statistics and Computing, 19:85-98, 2009.

[7] N.H. Bingham, C.M. Goldie and J.L. Teugels. Regular Variation, Cambridge University Press, 1987

[8] V. Chavez-Demoulin and A.C. Davison. Generalized additive modelling of sample extremes. Journal of the Royal Statistical Society, series C., 54:207-222, 2005.

[9] G. Collomb. Estimation non paramétrique de la régression par la méthode du noyau. $\mathrm{PhD}$ thesis, Université Paul Sabatier de Toulouse, 1976.

[10] A.C. Davison and N.I. Ramesh, N.I. Local likelihood smoothing of sample extremes. Journal of the Royal Statistical Society, series B, 62:191-208, 2000.

[11] A.C. Davison and R.L. Smith, R.L. Models for exceedances over high thresholds. Journal of the Royal Statistical Society, series B, 52:393-442, 1990.

[12] A. Dekkers and L. de Haan. On the estimation of the extreme-value index and large quantile estimation, Annals of Statistics, 17:1795-1832, 1989.

[13] J.H.J Einmahl. The empirical distribution function as a tail estimator, Statistica Neerlandica, 44:79-82, 1990.

[14] P. Embrechts, C. Klüppelberg, and T. Mikosch. Modelling extremal events, Springer, 1997.

[15] F. Ferraty and P. Vieu. Nonparametric modelling for functional data. Springer, 2005.

[16] A. Gannoun. Estimation non paramétrique de la médiane conditionnelle, médianogramme et méthode du noyau, Publications de l'Institut de Statistique de l'Université de Paris, XXXXVI:11-22, 1990.

[17] A. Gannoun, S. Girard, C. Guinot and J. Saracco. Reference ranges based on nonparametric quantile regression, Statistics in Medicine, 21:3119-3135, 2002.

[18] L. Gardes. Estimating the support of a Poisson process via the Faber-Shauder basis and extreme values. Publications de l'Institut de Statistique de l'Université de Paris, XXXXVI:4372,2002 .

[19] L. Gardes and S. Girard. A moving window approach for nonparametric estimation of the conditional tail index. Journal of Multivariate Analysis, 99:2368-2388, 2008.

[20] L. Gardes and S. Girard. Conditional extremes from heavy-tailed distributions: An application to the estimation of extreme rainfall return levels. Extremes, 13:177-204, 2010. 
[21] L. Gardes, S. Girard and A. Lekina. Functional nonparametric estimation of conditional extreme quantiles. Journal of Multivariate Analysis, 101:419-433, 2010.

[22] J. Geffroy. Sur un problème d'estimation géométrique. Publications de l'Institut de Statistique de l'Université de Paris, XIII:191-210, 1964.

[23] I. Gijbels and L. Peng. Estimation of a support curve via order statistics. Extremes, 3:251-277, 2000.

[24] S. Girard and P. Jacob. Extreme values and kernel estimates of point processes boundaries. ESAIM: Probability and Statistics, 8:150-168, 2004.

[25] S. Girard and P. Jacob. Frontier estimation via kernel regression on high power-transformed data. Journal of Multivariate Analysis, 99:403-420, 2008.

[26] S. Girard and L. Menneteau. Central limit theorems for smoothed extreme value estimates of point processes boundaries. Journal of Statistical Planning and Inference, 135(2):433-460, 2005.

[27] M.I. Gomes and M.J. Martins and M. Neves. Semi-parametric estimation of the second order parameter, asymptotic and finite sample behaviour. Extremes, 3:207-229, 2000.

[28] L. de Haan and A. Ferreira. Extreme Value Theory: An Introduction, Springer Series in Operations Research and Financial Engineering, Springer, 2006.

[29] P. Hall and N. Tajvidi. Nonparametric analysis of temporal trend when fitting parametric models to extreme-value data. Statistical Science, 15;153-167, 2000.

[30] P. Hall, M. Nussbaum, and S. Stern. On the estimation of a support curve of indeterminate sharpness. Journal of Multivariate Analysis, 62(2):204-232, 1997.

[31] W. Härdle, B. U. Park, and A. B. Tsybakov. Estimation of a non sharp support boundaries. Journal of Multivariate Analysis, 43:205-218, 1995.

[32] B.M. Hill. A simple general approach to inference about the tail of a distribution. The Annals of Statistics, 3:1163-1174, 1975.

[33] A.P. Korostelev and A.B. Tsybakov. Minimax theory of image reconstruction, volume 82 of Lecture Notes in Statistics. Springer-Verlag, New-York, 1993.

[34] L. Meligkotsidou, I. Vrontos and S. Vrontos. Quantile regression analysis of hedge fund strategies. Journal of Empirical Finance, 16:264-279, 2009.

[35] L. Menneteau. Multidimensional limit theorems for smoothed extreme value estimates of point processes boundaries. ESAIM: Probability and Statistics, 12:273-307, 2008. 
[36] J. Pickands. Statistical inference using extreme order statistics. The Annals of Statistics, 3:119-131, 1975.

[37] G.G. Roussas. Nonparametric estimation of the transition distribution function of a Markov process. Ann. Math. Statist., 40:1386-1400, 1969.

[38] T. Samanta. Non-parametric estimation of conditional quantiles. Statistics and Probability Letters, 7:407-412, 1989.

[39] R.L. Smith. Extreme value analysis of environmental time series: an application to trend detection in ground-level ozone (with discussion). Statistical Science, 4:367-393, 1989.

[40] C.J. Stone. Consistent nonparametric regression (with discussion). The Annals of Statistics, 5:595-645, 1977.

[41] W. Stute. Conditional empirical processes. The Annals of Statistics, 14:638-647, 1986.

[42] I. Weissman. Estimation of parameters and large quantiles based on the $k$ largest observations, Journal of the American Statistical Association, 73:812-815, 1978.

[43] Q. Yao. Conditional predictive regions for stochastic processes. Technical report, University of Kent at Canterbury, 1999. 


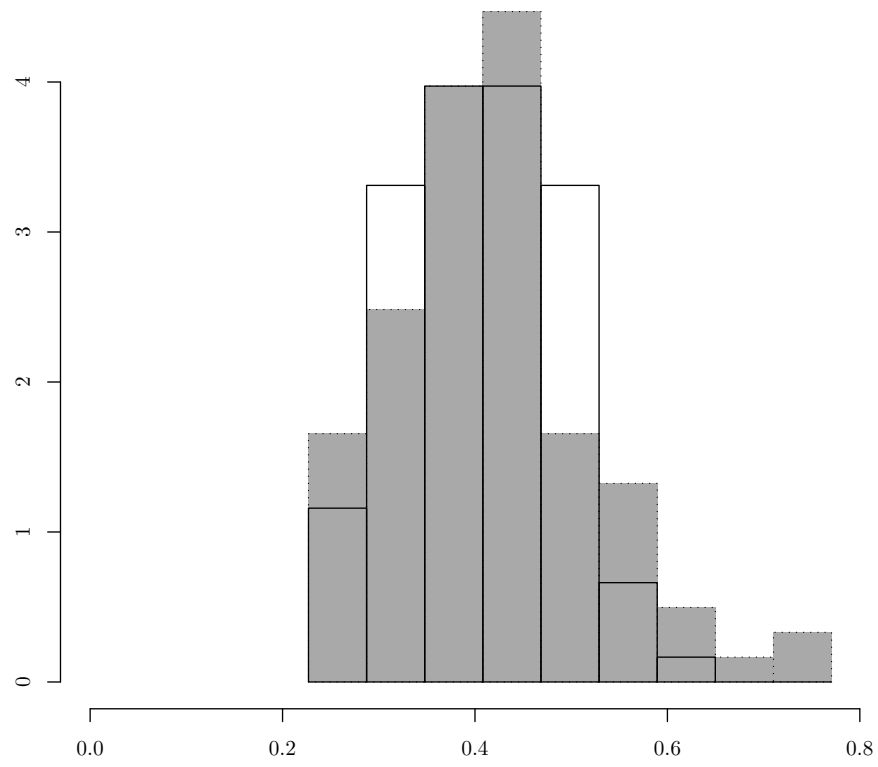

Figure 1: Comparison between the error distributions obtained with the cross-validation strategy $\left(\Delta_{c v}^{(p)}\right.$, light gray) and the oracle strategy $\left(\Delta_{\text {oracle }}^{(p)}\right.$, transparent) on $N=100$ samples of size $n=300$. 

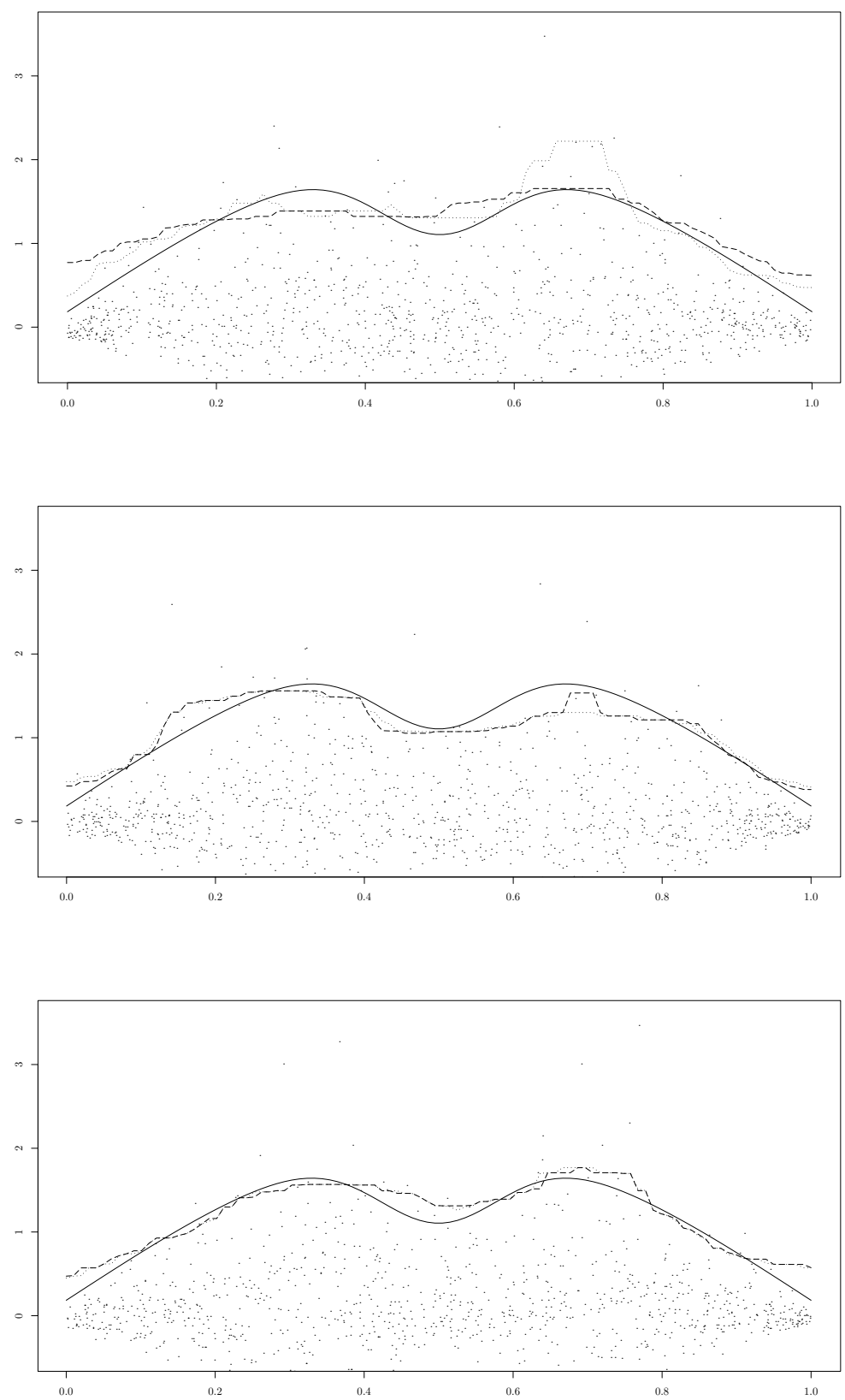

Figure 2: Comparison of the true quantile (solid line) with the estimated ones obtained by the cross-validation strategy (dotted line) and the oracle strategy (dashed line). The sample size is $n=1000$. The vertical axis is in a logarithmic scale. Top: worst $10 \%$ estimator, middle: median estimator, bottom: best $10 \%$ estimator. 

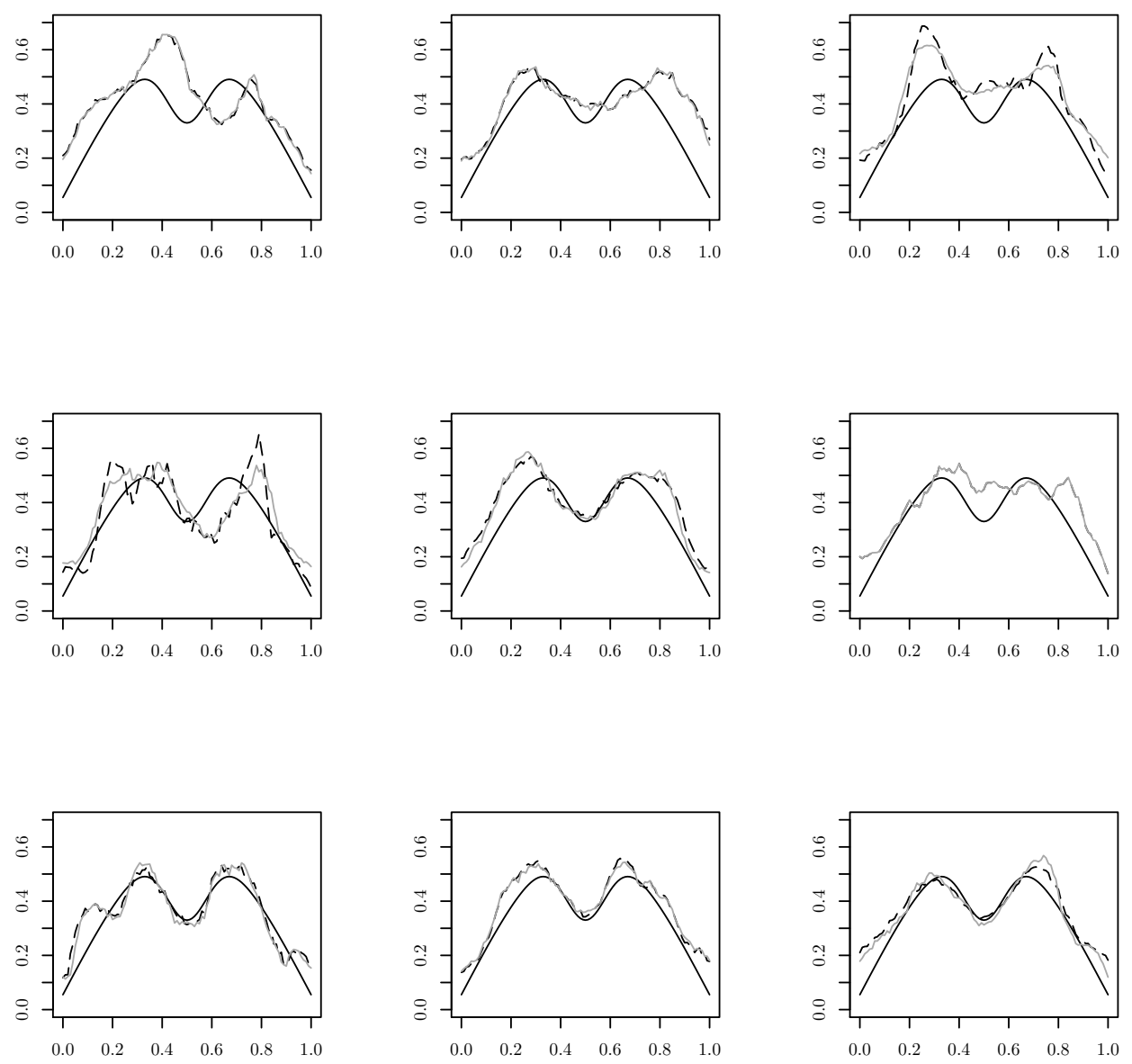

Figure 3: Comparison of the true tail index (solid line) with the Hill type estimator obtained by the cross-validation strategy (dotted line) and the oracle strategy (grey line). The sample size is $n=1000$. Top: worst $10 \%$ estimator, middle: median estimator, bottom: best $10 \%$ estimator. Left: $\alpha_{n}=0.2$, center: $\alpha_{n}=0.3$, right: $\alpha_{n}=0.4$. 


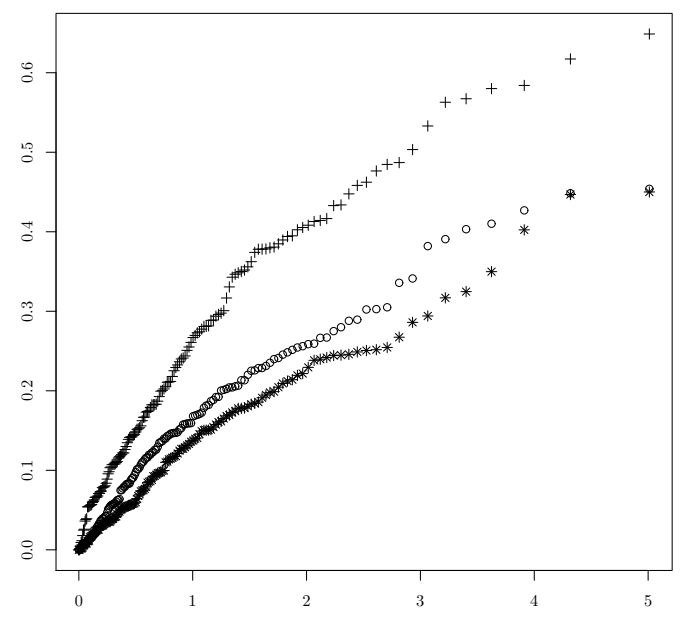

Figure 4: QQ-plots obtained at three different points: $x=0.25(\times \times \times), x=0.50(\circ \circ \circ)$ and $x=0.75(+++)$.

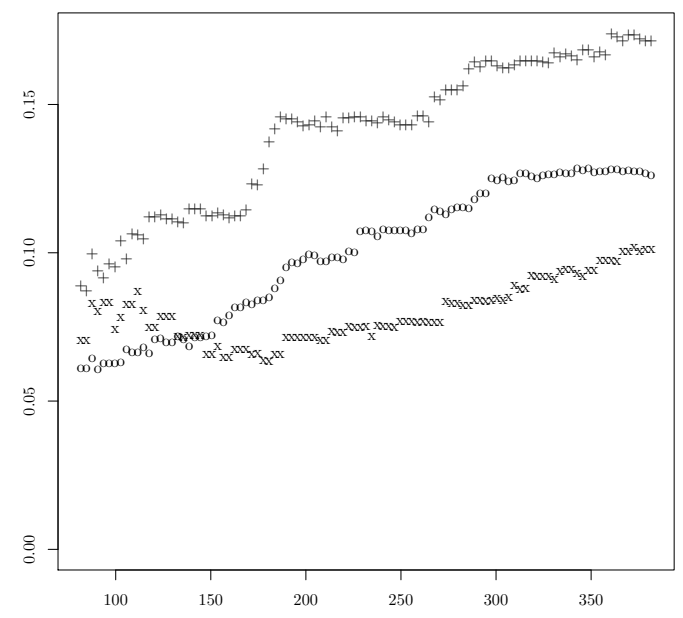

Figure 5: Hill plots obtained at three different points: $x=0.25(\times \times \times), x=0.50(\circ \circ \circ)$ and $x=0.75(+++)$. 


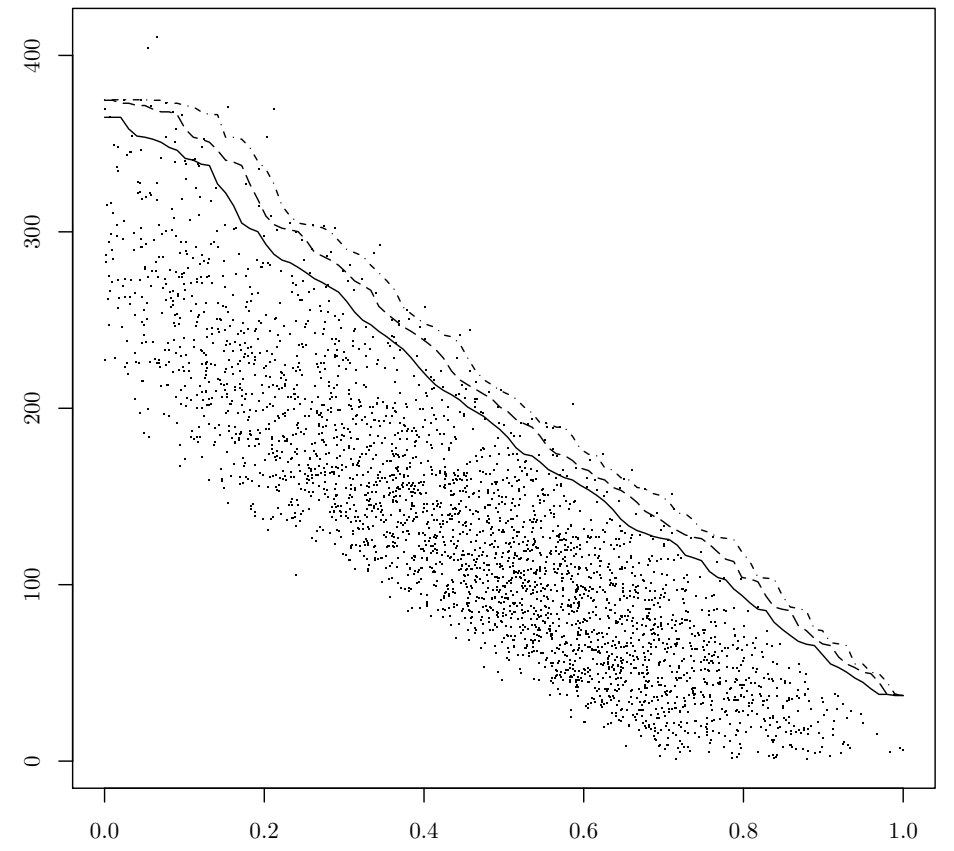

Figure 6: Real data scatter-plot $\left(X_{i}, Y_{i}\right), i=1, \ldots, n$ and estimated extreme level curves $(\zeta=20$ : solid line, $\zeta=10$ : dashed line, $\zeta=5$ : dash-dotted line) 